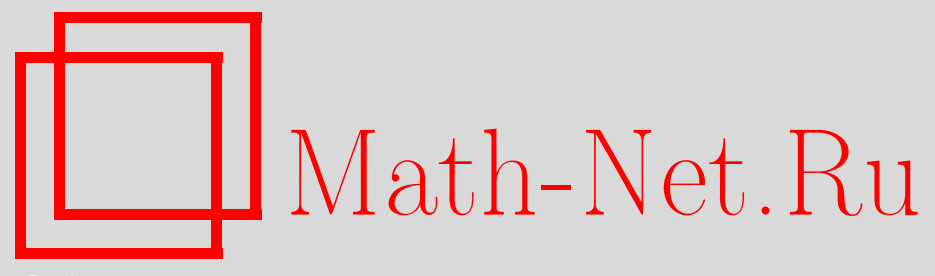

К. С. Колегов, А. И. Лобанов, Численное исследование массопереноса в капельно-пленочных системах с использованием регуляризованной разностной схемы в испарительной литографии, Вестн. Сам. гос. техн. ун-та. Сер. Физ.-мат. науки, 2018, номер 2, 344-363

DOI: https://doi.org/10.14498/vsgtu1601

Использование Общероссийского математического портала MathNet.Ru подразумевает, что вы прочитали и согласны с пользовательским соглашением

http://www.mathnet.ru/rus/agreement

Параметры загрузки:

IP : 54.210 .77 .194

26 апреля 2023 г., 14:03:03

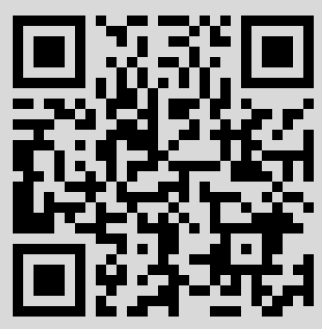


Вестн. Сам. гос. техн. ун-та. Сер. Физ.-мат. науки. 2018. Т. 22, № 2 . С. $344-363$ ISSN: 2310-7081 (online), 1991-8615 (print)

УДК 517.958:531.32

\title{
Численное исследование массопереноса в капельно-пленочных системах с использованием регуляризованной разностной схемы в испарительной литографии
}

\author{
К. С. Колегов ${ }^{1,2}$, А. И. Лобанов \\ 1 Астраханский государственный университет, \\ Россия, 414056, Астрахань, ул. Татищева, 20а. \\ 2 Волжский государственный университет водного транспорта \\ (Каспийский институт морского и речного транспорта филиал ФГБОУ ВО «ВГУВТ»), \\ Россия, 414000, Астрахань, ул. Никольская, 6. \\ 3 Московский физико-технический институт (государственный университет), \\ Россия, 141700, Московская обл., Долгопрудный, Институтский пер., 9.
}

\begin{abstract}
Аннотация
Массоперенос в высыхающих каплях и пленках интересен с практической точки зрения, так как применяется в задачах испарительной литографии. При создании условий неравномерного испарения с поверхности жидкого слоя возникают компенсационные потоки, перемещающие коллоидные частицы в области интенсивного испарения. Это позволяет получать на твердой поверхности микро- и наноструктуры требуемой формы. В работе описана нестационарная модель массопереноса в капельно-пленочных системах. Особенность модели заключается в совместном учете вязких, гравитационных и капиллярных сил. Для решения неустойчивой дискретной задачи о высыхающей капле (пленке) предложена регуляризованная разностная схема, на базе которой разработан комплекс программ. По результатам проведенных вычислительных экспериментов предложен способ получения кольцевых структур из микро- и наночастиц методом испарительной литографии.
\end{abstract}

Ключевые слова: массоперенос, капли, пленки, испарительная литография.

Получение: 10 января 2018 г. / Исправление: 15 мая 2018 г.

Принятие: 11 июня 2018 г. / Публикация онлайн: 27 июня 2018 г.

\section{Научная статья}

(2) () Контент публикуется на условиях лицензии Creative Commons Attribution 4.0 International (https://creativecommons.org/licenses/by/4.0/deed.ru)

\section{Образец для цитирования}

Колегов К. С., Лобанов А. И. Численное исследование массопереноса в капельнопленочных системах с использованием регуляризованной разностной схемы в испарительной литографии // Вестн. Сам. гос. техн. ун-та. Сер. Физ.-мат. науки, 2018. Т. 22, № 2. C. 344-363. doi: $10.14498 / v s g t u 1601$.

\section{Сведения об авторах}

Константин Сергеевич Колегов (1) http://orcid.org/0000-0002-9742-1308

младший научный сотрудник; лаб. математического моделирования и информационных технологий в науке и образовании ${ }^{1}$; старший преподаватель; каф. математических и естественнонаучных дисциплин ${ }^{2}$; e-mail: konstantin.kolegov@asu.edu.ru

\section{Алексей Иванович Лобанов (1) http://orcid.org/0000-0003-0391-3289}

доктор физико-математических наук; профессор; каф. вычислительной математики ${ }^{3}$;

e-mail: alexey@crec.mipt.ru 
Введение. Интерес исследователей к изучению процессов дегидратационной самоорганизации не угасает на протяжении последних двух или более десятилетий. Явления, происходящие в процессе высыхания капель и пленок, описаны, например, в $[1,2]$. Обычно при высыхании возникают течения жидкости, вызванные испарением. Они приводят к конвекционному переносу коллоидных частиц. Использование таких течений лежит в основе испарительной литографии [3], производства микрофлюидных чипов [4], создания открытых реакторов [5].

При изучении эффекта «кофейных колец» была предложена теория [6], согласно которой можно управлять процессом осаждения частиц во время высыхания капли, манипулируя концентрацией пара вблизи двухфазной границы. На основании данной теории разработан метод, получивший название «испарительная литография» [3]. Его суть заключается в создании условий для неравномерного испарения с поверхности коллоидной жидкости. В результате возникают компенсационные потоки, которые переносят взвешенные частицы в области интенсивного испарения. Управлять плотностью потока пара вдоль свободной поверхности жидкого слоя можно, к примеру, размещая над каплей маску [3]. Это своего рода «шаблон», так как получаемая после высыхания структура осажденных на подложку частиц повторяет форму отверстий в маске.

В настоящее время существует множество математических моделей, описывающих массоперенос в высыхающих каплях и пленках. Однако они обладают несколькими недостатками.

Во-первых, в таких моделях, как правило, не учитывается влияние силы тяжести. В [6,7] рассматриваются капли капиллярного размера. Так как в таких открытых системах капиллярные силы доминируют над гравитационными, последними зачастую пренебрегают. В этом случае форма поверхности капель близка к форме сферического сегмента. Для капли в форме сферического сегмента давление в ней постоянно во всем объеме. При отсутствии испарения капля находится в равновесии с внешней средой. Значительное отклонение от равновесной формы заметно лишь, когда капиллярное течение не успевает компенсировать быстрое испарение жидкости [7]. Равновесная форма капель, размер которых превышает капиллярную длину, далека от формы сферического сегмента [8]. Вдали от линии контакта с горизонтальной подложкой поверхность практически плоская. В [8] описаны результаты экспериментального исследования формы капель в зависимости от силы тяжести. Математическому моделированию зависимости формы капли от силы тяжести посвящена [9]. Расчеты [9] показали, что капля постоянного объема при увеличении ускорения свободного падения растекается сильнее, высота ее уменьшается, а поверхность уплощается. В [10] приводится сравнение экспериментального профиля (для разных капель) с аппроксимацией поверхности сферическим сегментом. При исследовании испарения жидкости в открытой цилиндрической ячейке силу тяжести также зачастую не принимают во внимание. Считается, что ячейка достаточно мала и объемные силы не влияют на форму границы раздела фаз жидкости и газа. Например, в [11] определяется поле скоростей течений в ячейке для частного случая, когда поверхность жидкости плоская. Модель [5] не объясняет причины возникновения течения и факторы, влияющие на форму границы «жидкость-газ». 
Во-вторых, чаще всего используется квазистационарный подход в совокупности с приближением смазки [7,18,26-28]. У этого метода есть недостатки. Он подходит для описания динамики жидкости лишь в тонких каплях ${ }^{1}$ и в случае медленного испарения [14]. Существует несколько нестационарных моделей, но они в основном посвящены чистым жидкостям [29, 30].

В-третьих, в большинстве случаев не учитывается возможность наличия маски над каплей или пленкой [26, 28, 31,32]. Кинематическая модель [33] не объясняет причины возникновения течений в пленке коллоидного раствора, сохнущей под маской.

Поэтому целью данной работы является разработка нестационарной модели массопереноса в испаряющихся каплях и пленках, которая учитывает влияние вязких, капиллярных и гравитационных сил, а также наличие маски в системе.

1. Физическая постановка задачи. Рассматриваются два случая. В первом капля покоится на горизонтальном непроницаемом основании в режиме закрепленной трехфазной границы «жидкость-газ-подложка» (пиннинг). Во втором случае жидкость находится в открытой цилиндрической ячейке.

Для описания процессов в каплях удобно использовать цилиндрические координаты, как и в [12-15]. Ось z направлена по нормали к твердому основанию в центре капли (пленки). Подложке (дну ячейки) соответствует $z=0$. Считаем, что толщина жидкого слоя мала. Высота тонкой капли (пленки) много меньше диаметра основания (ячейки), поэтому ограничимся рассмотрением одномерной модели с осредненными по глубине капли величинами.

В коллоидных растворах существенными являются эффекты диффузии, полимеризации, седиментации и переноса течением частиц, а также их адгезия к подложке и стенкам сосуда. В системе могут происходить различные фазовые переходы: парообразование, формирование поверхностной пленки из взвешенного и растворенного в жидкости вещества, «золь-гель»-переход, кристаллизация в солевом растворе и так далее.

Процессы термоконвекции и гравитационные течения подробно исследованы средствами численного моделирования в [16]. Здесь наше внимание уделяется течению компенсационной природы. Считаем, что тонкий слой чистого растворителя или разбавленного раствора испаряется медленно. В таком случае термоконвекцией допустимо пренебречь.

Концентрация частиц достаточно мала, поэтому плотность раствора $\rho$ считаем постоянной, а жидкость - несжимаемой. Пусть плотности вещества частиц и жидкости приблизительно равны между собой. Частицы в таком растворе продолжительное время находятся во взвешенном состоянии, поэтому седиментацией пренебрегаем. Также не рассматривается адгезия частиц к твердой поверхности. Считается, что для разбавленного раствора при малом градиенте температуры (из-за медленного испарения) вязкость $\eta$, коэффициенты поверхностного натяжения $\sigma$ и диффузии $D$ постоянны. Например, по экспериментальным данным [17], вязкость меняется незначительно при повышении массовой доли полистирольных частиц вплоть до значения 0.3. Здесь рассматривается начальная стадия процесса (гидродинамический этап) задолго до формирования геля.

\footnotetext{
${ }^{1}$ Капли, высота которых много меньше радиуса основания.
} 


\section{2. Основные уравнения нестационарной математической модели.} Приведем уравнения нестационарной модели, описывающей массоперенос в испаряющейся жидкости [12-15], в безразмерной форме. Система включает закон сохранения массы

$$
\frac{\partial h}{\partial t}+\frac{1}{r} \frac{\partial(r h u)}{\partial r}=-J l
$$

и уравнение движения

$$
\frac{\partial u}{\partial t}+u \frac{\partial u}{\partial r}=-\frac{\partial P}{\partial r}+\frac{1}{r} \frac{\partial}{\partial r}\left(r \frac{\partial u}{\partial r}\right)-\frac{u}{r^{2}}+\frac{r}{h} \frac{\partial h}{\partial r} \frac{\partial}{\partial r}\left(\frac{u}{r}\right)-\operatorname{Ar} \frac{\partial h}{\partial r}
$$

Для случая коллоидной жидкости в систему добавляется уравнение конвекции-диффузии

$$
\frac{\partial c}{\partial t}+u \frac{\partial c}{\partial r}=\frac{1}{\operatorname{Pe}} \frac{1}{r h} \frac{\partial}{\partial r}\left(r h \frac{\partial c}{\partial r}\right)+\frac{J c l}{h} .
$$

В (1)-(3) $h$ - толщина слоя жидкости, $u$-осредненная по глубине слоя радиальная скорость течения, $J$ - плотность потока пара, $P$ - давление, $c-$ массовая доля коллоидных частиц в растворе, $l=\sqrt{1+(\partial h / \partial r)^{2}}$. Безразмерные критерии подобия вычисляются по характерным размерным параметрам задачи. Число Пекле $\mathrm{Pe}=U_{c} L_{c} / D=\eta /(\rho D)$, характерная скорость $U_{c}=\eta /\left(\rho L_{c}\right)$, характерный размер $L_{c}=V^{1 / 3}, V$ - объем капли. Число Архимеда $\mathrm{Ar}=g L_{c}^{3} \rho^{2} / \eta^{2}, g$ - ускорение свободного падения. Вывод уравнений модели описан в [15].

Выведем вид замыкающего соотношения, играющего роль уравнения состояния. Давление можно определить как давление Лапласа $\sigma\left(1 / R_{1}+1 / R_{2}\right)$. Здесь $R_{1}$ и $R_{2}$ - радиусы кривизны. В итоге имеем уравнение состояния в безразмерном виде

$$
P=-\frac{1}{\mathrm{Ca}}\left(\frac{1}{l^{3}} \frac{\partial^{2} h}{\partial r^{2}}+\frac{1}{r l} \frac{\partial h}{\partial r}\right)
$$

где $\mathrm{Ca}=\eta^{2} /\left(\rho \sigma L_{c}\right)$ - капиллярное число.

\section{3. Сравнение двух моделей течений в испаряющейся жидкости.}

3.1. Квазистационарный подход. Одномерная квазистационарная модель динамики испаряющейся жидкости представляет собой упрощение модели Фишера [7]. В системе уравнений модели [7] опущены слагаемые с $\partial u / \partial t$.

3.1.1. Уравнения модели. Подход Фишера [7] базируется на приближении смазки и результатах [18]. Модельные уравнения [7] - упрощенная система уравнений Навье-Стокса, когда аспектное соотношение мало, $\varepsilon=h_{0} / R \ll 1$, где $h_{0}=h(0,0)$. Для капли с краевым углом $\theta \rightarrow 0$ по теории смазки $l \approx 1$. Поэтому уравнение (1) принимает вид

$$
\frac{\partial h}{\partial t}+\frac{1}{r} \frac{\partial(r h u)}{\partial r}=-J
$$

Уравнение состояния (4) упрощается и принимает вид

$$
P=-\frac{1}{\mathrm{Ca}} \frac{1}{r} \frac{\partial}{\partial r}\left(r \frac{\partial h}{\partial r}\right)
$$


Для радиальной скорости с учетом (6) имеем

$$
u=\frac{1}{\text { Ca }} \frac{\partial}{\partial r}\left(\frac{1}{r} \frac{\partial}{\partial r}\left(r \frac{\partial h}{\partial r}\right)\right) \frac{h^{2}}{3} .
$$

Введем вспомогательную величину - производную глубины жидкого слоя

$$
\omega=-\frac{1}{\mathrm{Ca}} \frac{\partial h}{\partial r}
$$

Тогда (7) можно переписать в виде

$$
u=-\frac{\partial}{\partial r}\left(\frac{1}{r} \frac{\partial(r \omega)}{\partial r}\right) \frac{h^{2}}{3}
$$

3.1.2. Краевые условия. В силу осевой симметрии

$$
\left.\frac{\partial h(r, t)}{\partial r}\right|_{r=0}=0, \quad u(0, t)=0 .
$$

Для точки трехфазной границы в силу прилипания $h(R, t)=0, u(R, t)=0$. Начальное условие для формы капли $h(r, 0)=\varepsilon R\left(1-r^{2} / R^{2}\right)$.

При рассмотрении жидкости в цилиндрической ячейке считается, что жидкость зафиксирована у верхней границы стенки ячейки (например, как в эксперименте [5]). Используются краевые условия $h(R, t)=h(r, 0)=\varepsilon R$, так как здесь $h_{0}-$ не только начальная толщина жидкого слоя, но и высота ячейки.

3.2. Нестационарный подход.

3.2.1. Преобразование уравнений модели. Введем вспомогательную величину - скорость сдвига

$$
\dot{\gamma}=\frac{\partial u}{\partial r}
$$

тогда с учетом (6), (8) и (10) уравнение движения (2) приводится к следующему виду:

$$
\frac{\partial u}{\partial t}+\frac{\partial}{\partial r}\left(\frac{1}{r} \frac{\partial(r \omega)}{\partial r}\right)-\frac{\partial \dot{\gamma}}{\partial r}=\left(\frac{\mathrm{Ca} \omega r}{h}-1\right) \frac{u}{r^{2}}-\left(u-\frac{1}{r}+\frac{\mathrm{Ca} \omega}{h}\right) \dot{\gamma}
$$

В (9) и (11) не учтена сила тяжести (отсутствует слагаемое с числом Архимеда), так как в [7] численно исследуется динамика лишь в микрокаплях.

3.2.2. Краевые и начальные условия. Кроме граничных и начальных условий из п. 3.1.2, для нестационарной модели необходимо поставить начальное условие для скорости течения. Считаем, что в начальный момент жидкость покоилась, $u(r, 0)=0$. Запишем еще одно граничное условие в центре:

$$
\left.\frac{\partial \dot{\gamma}(r, t)}{\partial r}\right|_{r=0}=0
$$

так как при $r \rightarrow 0 u \sim r[15]$. 
3.3. Плотность потока пара. Плотность потока пара может изменяться в зависимости от условий окружающей среды (атмосферное давление, температура, влажность и так далее). Для случая капли на подложке сравнение двух моделей проведем на примере модельного закона испарения [7]

$$
J=\frac{1-\exp \left(-A(r-R)^{2}\right)}{K+h} E,
$$

где $E, K$ и $A$-параметры. Число $A$ задает темп убывания $J$ вблизи трехфазной границы, неравновесный параметр $K$ определяет разность скорости испарения в центре капли и вблизи периферии $(K \rightarrow 0$ в случае быстроиспаряющейся жидкости и $K \rightarrow \infty$ для нелетучей жидкости), число испарения $E$ характеризует интенсивность перехода жидкости в пар. Модельный закон испарения (12) выбран по причине того, что при его использовании $J(R, t)=0$. Это согласуется со следствием из закона сохранения массы (5) при заданных граничных условиях для капли (п. 3.1.2).

При описании испарения жидкости из ячейки воспользуемся модельным законом [19]

$$
J=\frac{1-\exp \left(-m \sqrt{1-r^{2} / R^{2}}\right)}{(1-\exp (-m)) \sqrt{1-r^{2} / R^{2}}} E,
$$

где $m-$ положительное число. В данном случае из $(5)$ следует, что $J(R, t) \neq 0$, так как $h(R, t) \neq 0$.

3.4. Результаты расчета. Нестационарная система уравнений (5), (8), (10), (11), а также квазистационарная система уравнений (5), (8) с замыкающим соотношением (9) решались численно (см. п. 6). Параметры расчетов приведены в табл. 1.

Эксперименты $[5,20]$ показали, что на финальной стадии, когда слой жидкости в центральной части становится примерно в 30-40 раз тоньше, радиальная скорость течения увеличивается примерно на порядок. Для нестационарной модели капли воды на подложке максимальная скорость составляет примерно $45 \mathrm{mкм} / \mathrm{c}(t \approx 1.5 \mathrm{мин})$, что хорошо согласуется с экспериментом [20]

Таблица 1

Физические свойства жидкостей (при температуре $20^{\circ} \mathrm{C}$ ) и параметры задачи [Тhе physical properties of liquids (at temperature $20^{\circ} \mathrm{C}$ ) and the parameters of the problem]

The physical properties of liquids

вязкость [viscosity], $\eta(\mathrm{cPa})$

плотность [density], $\rho\left(\mathrm{kg} / \mathrm{m}^{3}\right)$

коэффициент поверхностного натяжения

[surface tension coefficient], $\sigma(\mathrm{N} / \mathrm{m})$

радиус основания капли

[radius of the base of the drop], $R(\mathrm{~m})$

аспектное соотношение [aspect ratio], $\varepsilon=h_{0} / R$

капиллярное число [capillary number], Са

The parameters of the problem for

\begin{tabular}{l|c} 
water & $\begin{array}{c}\text { ethylene } \\
\text { glycol }\end{array}$ \\
\hline
\end{tabular}

$20 \cdot 10^{-3}$

1113

998

$46.1 \cdot 10^{-3}$

$72.8 \cdot 10^{-3}$

$0.1 \cdot 10^{-3}$

$0.6 \cdot 10^{-3}$

0.06

0.3

0.14 
(рис. 1, а). Квазистационарная модель дает финальное значение скорости около $35 \mathrm{mкм} / \mathrm{c}$ (рис. 1, а). Отметим, что наибольшая погрешность измерений в [20] в конце процесса составляет $\pm 28 \%$. Погрешность численных расчетов не превышает $2 \%$. В случае с открытой цилиндрической ячейкой, заполненной этиленгликолем, рассчитанные значения скоростей с помощью двух моделей также расходятся на финальной стадии испарения $(t \approx 2.34$ мин, рис. $1, b)$. Наибольшие скорости составляют примерно 0.5 и 0.7 мкм/с при расчетах по квазистационарной и нестационарной моделям соответственно (рис. $1, b)$. На рисунках знаком «тильда» обозначены безразмерные величины. В [5] скорость течения возрастала приблизительно от $0.1 \mathrm{mKM} / \mathrm{c}$ до $1 \mathrm{MKM} / \mathrm{c}$ в конце процесса. Погрешность измерений в [5] не указана. Экспериментальный метод [5] не позволил авторам получить достоверные данные вблизи оси симметрии на позднем временном этапе из-за малого количества микрочастиц, находящихся в этой области (большую часть микрочастиц к этому времени снесло течением к стенке ячейки). Таким образом, по экспериментальным данным [5] сложно определить, в какой точке скорость достигла максиму-

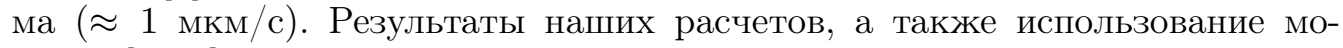
делей $[5,11]$ показывают, что максимальное значение скорости достигается вблизи центра ячейки, $r \in[0.1 R, 0.3 R]$.
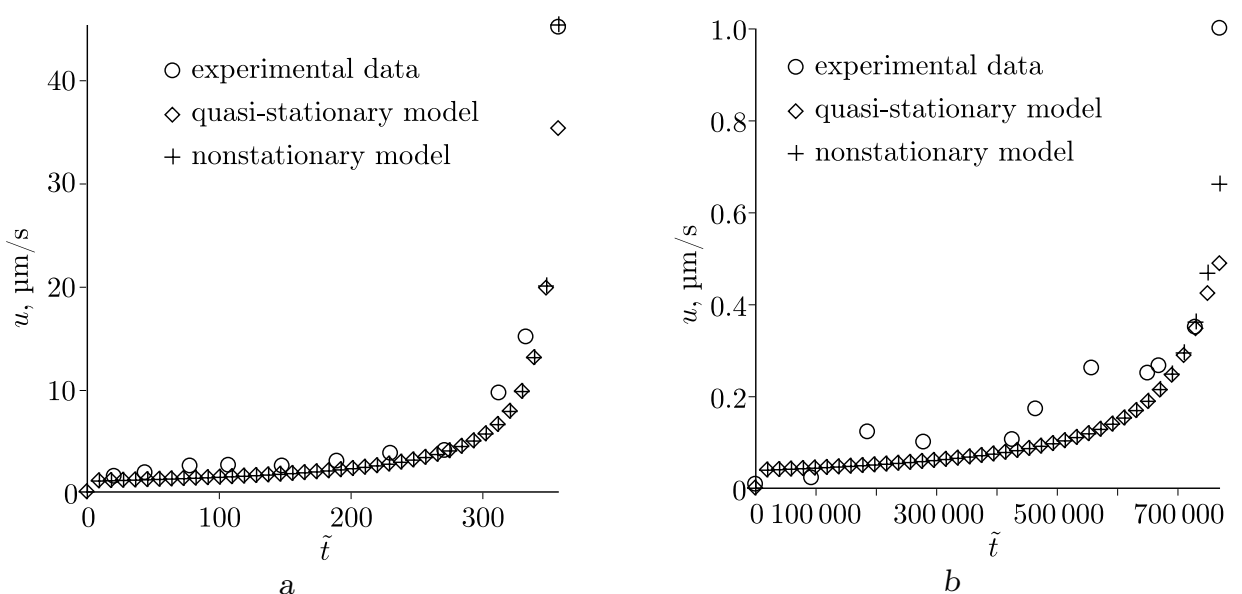

Рис. 1. Сравнение результатов расчета скоростей течений с экспериментальными данными $[5,20]:(\mathrm{a})$ вода $(r \approx 0.7 R, s \approx 1.2$ для квазистационарной модели, среднеквадратичное отклонение $s \approx 0.47$ для нестационарной модели); $(b)$ этиленгликоль $(r \approx 0.2 R, s \approx 0.0526$ для квазистационарной модели, среднеквадратичное отклонение $s \approx 0.0358$ для нестационарной модели). Параметры модельного закона испарения: (а) в (12) $E=1.1 \cdot 10^{-4}$,

$$
K=0.0985, A=100 ;(b) \text { в }(13) E=0.575 \cdot 10^{-7}, m=1
$$

[Figure 1. Comparison of the results of calculation of flow velocities with experimental data [5,20]: (a) water $(r \approx 0.7 R, s \approx 1.2$ for quasi-stationary model, standard deviation $s \approx 0.47$ for nonstationary model); $(b)$ ethylene glycol $(r \approx 0.2 R, s \approx 0.0526$ for quasi-stationary model, standard deviation $s \approx 0.0358$ for nonstationary model). Parameters of the model evaporation law: (a) in (12) $E=1.1 \cdot 10^{-4}, K=0.0985, A=100$; (b) in (13) $E=0.575 \cdot 10^{-7}, m=1$ ]

3.5. Вывод. Результаты расчетов по двум моделям хорошо согласуются с экспериментальными данными. Различие значений скорости при расчетах по нестационарной и квазистационарной моделям существенно лишь на финальной стадии. Значение среднеквадратичного отклонения от эксперимен- 
тальных данных при расчете по нестационарной модели примерно в два раза меньше соответствующего значения для квазистационарной модели.

Согласно экспериментальным [5,20] и расчетным данным, скорости испарения и радиального течения жидкости зависят от ее вязкости. На примере этиленгликоля и воды показано, что скорости тока жидкости в подобных системах могут различаться на порядок.

\section{4. Моделирование массопереноса в коллоидном растворе, испа- ряющемся из цилиндрической ячейки под маской в форме концен- трических колец.}

4.1. Образование кольцевых структур в результате дегидратационной самоорганизации. Литография, основанная на испарении, является одним из перспективных методов формирования структур из микро- и наночастиц [3]. Испарение из пленки или капли коллоидного раствора становится неравномерным, например, из-за размещения над жидкостью маски. В результате возникает течение компенсационной природы, которое сносит частицы к заданным шаблоном (маской) местам. Шаблоном может служить металлический цилиндр, стекловолокно [21] или пластинка с равномерно расположенными отверстиями [3]. Характер испарения, зависящий от вида шаблона, определяет форму получаемой структуры. В [12] предложен способ формирования структур в виде концентрических колец из микро- и наночастиц, основанный на методе испарительной литографии. Для тестовых расчетов ранее использовалась упрощенная модель [12], не учитывающая влияние вязких и гравитационных сил. Сверху ячейка закрывается специальной маской (рис. 2, а), которая состоит из колец, скрепленных между собой относительно тонкими и достаточно крепкими перемычками (рис. $2, b)$. В качестве примера на рис. $2, b$ изображен шаблон с четырьмя кольцевыми отверстиями.

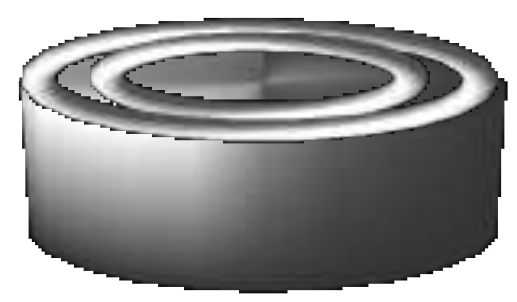

a

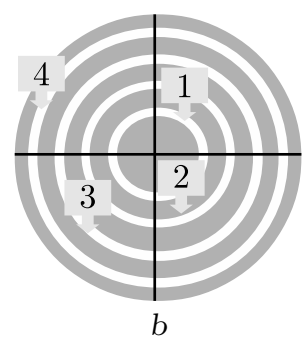

Рис. 2. (a) ячейка закрытая маской; $(b)$ шаблон из концентрических колец [Figure 2. The cell (a) is closed by the mask (b) from concentric rings

4.2. Математическая модель. С учетом (4) и (8) уравнение, описывающее зависимость капиллярного давления, записывается как

$$
P=\frac{1}{l^{3}} \frac{\partial \omega}{\partial r}+\frac{\omega}{r l}
$$

где $l=\sqrt{1+(\mathrm{Ca} \omega)^{2}}$.

Уравнение (2) с учетом (4), (8) и (10) записывается в виде

$$
\frac{\partial u}{\partial t}+\frac{\partial}{\partial r}(P-\dot{\gamma})=\text { Bo } \omega+\left(\frac{\mathrm{Ca} \omega r}{h}-1\right) \frac{u}{r^{2}}-\left(u-\frac{1}{r}+\frac{\mathrm{Ca} \omega}{h}\right) \dot{\gamma}
$$


Здесь Во = Са Ar - число Бонда. Система уравнений приведена к виду (1), (3), (8), (10), (14), (15).

Считаем, что плотность потока пара зависит лишь от препятствия, расположенного над жидкостью. Тогда

$$
J=\frac{1-\cos (2 \pi r N / R)}{2} E,
$$

где $N$ - количество кольцевых отверстий в шаблоне. Модельный закон (16) качественно согласуется с расчетами [3].

4.3. Краевые условия. К условиям (см. пп. 3.1.2 и 3.2.2)

$$
\begin{gathered}
\left.\frac{\partial h(r, t)}{\partial r}\right|_{r=0}=0, \quad u(0, t)=0 \\
u(R, t)=0, \quad u(r, 0)=0,\left.\quad \frac{\partial \dot{\gamma}(r, t)}{\partial r}\right|_{r=0}=0
\end{gathered}
$$

необходимо добавить еще несколько условий. На границе у стенки ячейки возможны два варианта - постоянная толщина жидкого слоя (трехфазная граница «жидкость-стенка-газ» закреплена) и постоянный краевой угол $\theta$. В первом случае для микроячейки выполняется [5]

$$
h(R, t)=h(r, 0)=\varepsilon R .
$$

Во втором случае для макроячейки

$$
\begin{gathered}
\left.\frac{\partial h(r, t)}{\partial r}\right|_{r=R}=\operatorname{ctg} \theta, \\
h(r, 0)=H(r) .
\end{gathered}
$$

Вид функции $H$ найдем из уравнения

$$
\left(r H^{\prime}\right)^{\prime}-\text { Bo } r H+C r=0,
$$

которое получено следующим образом (здесь штрихом обозначена производная $d H / d r)$. В начальный момент времени $\partial P / \partial r+\operatorname{Ar} \partial h / \partial r=0$. Интегрируем левую и правую части этого выражения, учитывая (4). Считаем, что в начальный момент времени кривизна мениска мала, $1+\left(H^{\prime}\right)^{2} \approx 1$.

Найдем константу $C$, проинтегрируем (20) от 0 до $R$ и умножим на $2 \pi / R^{2}$ :

$$
\frac{2 \pi}{R^{2}} \int_{0}^{R}\left(r H^{\prime}\right)^{\prime} d r-\frac{\mathrm{Bo}}{R^{2}} \int_{0}^{R} 2 \pi r H d r+\int_{0}^{R} C r d r=0
$$

Слагаемое

$$
\int_{0}^{R} 2 \pi r H d r=V / L_{c}^{3}=1
$$

при выбранном масштабе длины $L_{c}$. Тогда с учетом граничного условия (18) получаем

$$
C=-\frac{2}{R} \operatorname{ctg} \theta+\frac{\text { Bo }}{\pi R^{2}}
$$


При такой константе $C$ решением (20) является функция

$$
H(r)=-2 \frac{\operatorname{ctg} \theta}{\operatorname{Bo} R}+\frac{1}{\pi R^{2}}+\frac{I_{0}(r \sqrt{\mathrm{Bo}}) \operatorname{ctg} \theta}{\sqrt{\mathrm{Bo}} I_{1}(R \sqrt{\mathrm{Bo}})},
$$

где $I_{0}, I_{1}$ - модифицированные функции Бесселя нулевого и первого порядков соответственно. Отметим, что полученное выражение является лишь приближением к начальной равновесной форме. Найдем значение краевого угла при заданном радиусе ячейки. На границе у стенки ячейки $H(R)=\varepsilon R$, тогда

$$
\varepsilon R=-2 \frac{\operatorname{ctg} \theta}{\mathrm{Bo} R}+\frac{1}{\pi R^{2}}+\frac{I_{0}(R \sqrt{\mathrm{Bo}}) \operatorname{ctg} \theta}{\sqrt{\mathrm{Bo}} I_{1}(R \sqrt{\mathrm{Bo}})} .
$$

Выражение для краевого угла примет вид

$$
\theta=\operatorname{arcctg}\left(\left(\varepsilon R-\frac{1}{\pi R^{2}}\right)\left(\frac{I_{0}(R \sqrt{\mathrm{Bo}})}{\sqrt{\mathrm{Bo}} I_{1}(R \sqrt{\mathrm{Bo}})}-\frac{2}{\mathrm{Bo} R}\right)^{-1}\right) .
$$

Остается задать краевые условия для массовой доли частиц. В силу осевой симметрии и непротекания через стенку ячейки

$$
\left.\frac{\partial c(r, t)}{\partial r}\right|_{r=0}=0, \quad \frac{c(R, t)}{R}+\left.\frac{\partial c(r, t)}{\partial r}\right|_{r=R}=0 .
$$

Начальное условие $c(r, 0)=C_{0}$, где $C_{0}$ - заданная константа.

4.4. Параметры задачи. Для расчетов выберем параметры, соответствующие раствору из воды и монодисперсных полистироловых частиц. Начальная массовая доля частиц $C_{0}=0.05$. При такой концентрации раствор находится в фазе золя продолжительное время [17]. Кроме того, частицы долгое время находятся во взвешенном состоянии. Из-за малой массы и размеров седиментацией частиц под действием силы тяжести можно пренебречь. Коэффициент диффузии частиц $D=2 \cdot 10^{-11} \mathrm{~m}^{2} / \mathrm{c}$ [17], тогда число Пекле $\mathrm{Pe}=5 \cdot 10^{4}$.

Рассмотрим случай, когда коллоидная жидкость испаряется из микроячейки. Пусть радиус основания ячейки $R=2$ мм, аспектное отношение $\varepsilon=0.25$. При таких размерах трехфазная граница закреплена у верхней границы стенки ячейки [5]. Считаем, что начальная форма поверхности жидкости плоская [5]. Тогда объем жидкости $V=\pi \varepsilon R^{3}$. Получаем значения характерных величин: длина $L_{c} \approx 1.84 \mathrm{мм}$, скорость $U_{c} \approx 0.542 \mathrm{Mm} / \mathrm{c}$ и время $T_{c} \approx 3.4$ с. Капиллярное число и критерий Бонда принимают значения Са $\approx 7 \cdot 10^{-6}$ и Во $\approx 0.46$.

При рассмотрении жидкости в макроячейке вместо (17) должно выполняться условие постоянного угла смачивания (18) и начальное условие (19). Если $\theta>\pi / 2$ (силы притяжения жидкости и стенки емкости меньше сил притяжения между молекулами жидкости), то поверхность жидкости принимает форму выпуклого мениска, а при $\theta<\pi / 2$ (силы притяжения между молекулами жидкости и стенки ячейки больше сил притяжения между молекулами жидкости) - вогнутого. Пусть $R=16$ мм, $\varepsilon=0.25$, а объем жидкости составляет $90 \%$ от емкости ячейки $\left(V=0.9 \pi \varepsilon R^{3}\right)$. Получаем значения характерных 
величин $L_{c} \approx 14.3 \mathrm{мm}, U c \approx 70 \mathrm{mkм} / \mathrm{c}, T_{c} \approx 203$ с и безразмерных параметров $\mathrm{Ca} \approx 10^{-6}$, Bo $\approx 27$.

4.5. Результаты расчетов. Результаты численного решения системы уравнений (1), (3), (8), (10), (14), (15) с замыкающим соотношением (16) представлены на рис. 3. На рис. 3, a, 3, с изображены графики для случая с микроячейкой, а на рис. $3, b, 3, d$ - для макроячейки.

На рис. 3 , а и 3, b форма поверхности жидкости различается, что объясняется отношением капиллярных и гравитационных сил (критерий Бонда). Для микроячейки преобладающими являются капиллярные силы, поэтому форма вогнутого мениска похожа на сферический сегмент. Свободная гра-
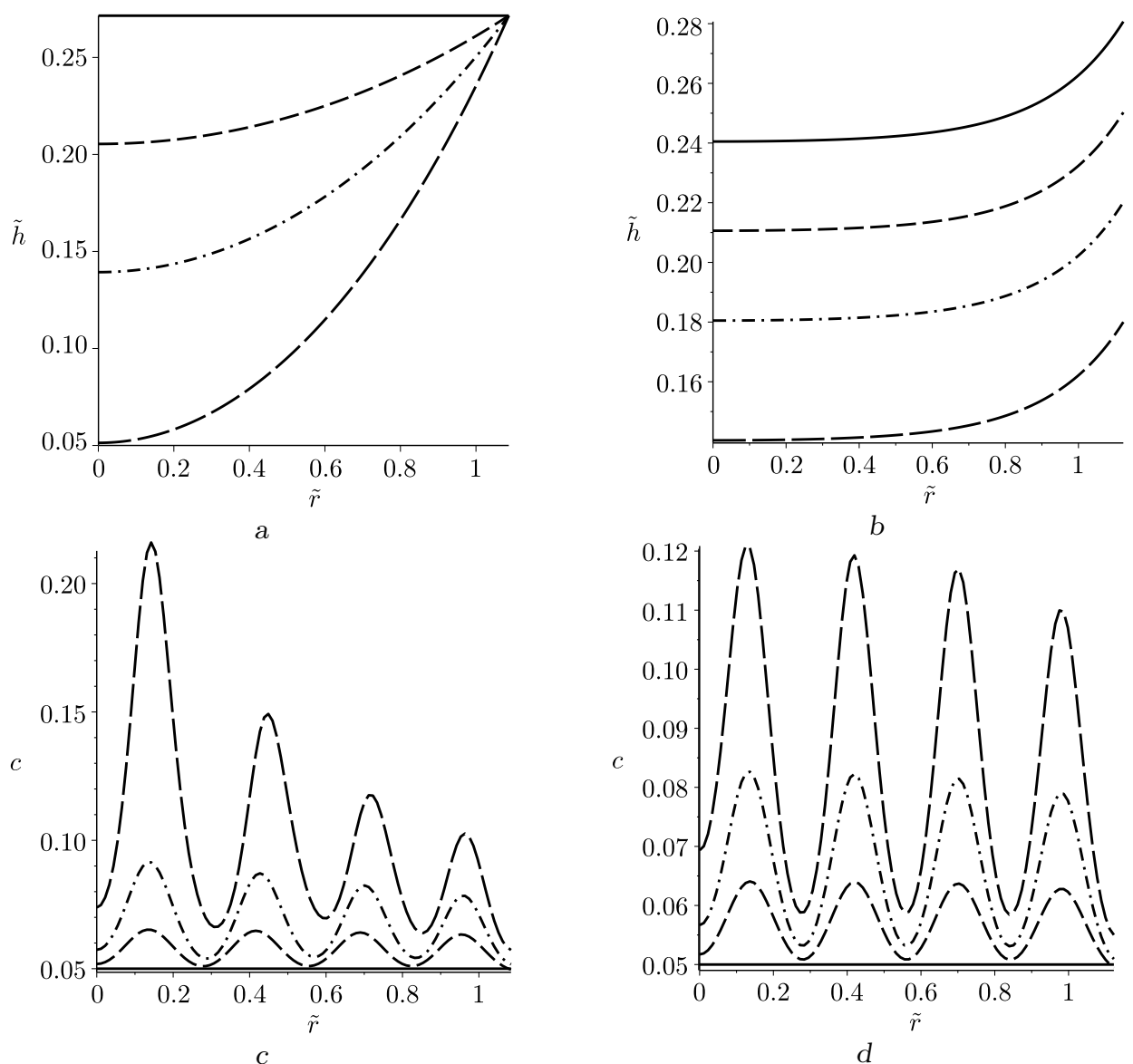

Рис. 3. Результаты расчета динамики коллоидной жидкости в микро- (слева) и макроячейке (справа) для времени $t=0,0.3 T, 0.6 T, T$ (для $(\mathrm{a}),(c) T \approx 340$ с и для $(b)$, (d) $T \approx 20307 \mathrm{c})$. Параметры расчета: $N=4 ; E=2.25 \cdot 10^{-3}$ для (a), (c) и $E=2 \cdot 10^{-3}$ для $(b)$, $(d)$. На рисунке приведены: $(a, b)$ толщина жидкого слоя, $(c, d)$ массовая доля коллоидных частиц

[Figure 3. The results of calculating the dynamics of a colloidal fluid in the micro- (left) and macrocell (right) for time $t=0,0.3 T, 0.6 T, T$ (for (a), (c) $T \approx 340 \mathrm{~s}$ and for $(b),(d)$ $T \approx 20307 \mathrm{~s})$. Calculation parameters: $N=4 ; E=2.25 \cdot 10^{-3}$ for (a), (c) and $E=2 \cdot 10^{-3}$ for $(b),(d)$. The figure shows: $(a, b)$ the thickness of the liquid layer, $(c, d)$ the mass fraction of colloidal particles] 
ница жидкости в макроячейке далека от формы сферического сегмента, что объясняется преобладанием силы тяжести.

В обоих случаях в процессе испарения массовая доля коллоидных частиц под кольцевыми отверстиями нарастает интенсивнее, чем в промежуточных зонах (рис. $3, c, 3, d$ ), что объясняется сносом этих частиц компенсационным течением.

\section{5. Моделирование массопереноса в испаряющейся капле колло- идного раствора.}

5.1. Основные уравнения и краевые условия. Система уравнений модели включает (1), (3), (8), (10), (14) и (15) с замыкающим соотношением (12). Начальные и граничные условия такие же, как в предыдущем разделе (см. п. 4.3). Исключение составляет граничное условие на краю для массовой доли частиц. Вместо условия непротекания $r^{-1} \partial(r c) / \partial r=0$ записывается условие $\partial c / \partial r=0$ при $r=R$, что следует из $(3)$, когда $h$ и $J$ равны нулю. Для случая с каплей решением (20) является функция

$$
H(r)=2 \frac{\operatorname{tg} \theta}{\mathrm{Bo} R}+\frac{1}{\pi R^{2}}-\frac{I_{0}(r \sqrt{\mathrm{Bo}}) \operatorname{tg} \theta}{\sqrt{\mathrm{Bo}} I_{1}(R \sqrt{\mathrm{Bo}})},
$$

где

$$
R= \begin{cases}\left(\frac{4}{\pi \operatorname{tg} \theta}\right)^{1 / 3}+\frac{\mathrm{Bo}}{18 \pi \operatorname{tg} \theta}-\frac{\mathrm{Bo}^{2}}{81 \cdot 2^{11 / 3}(\pi \operatorname{tg} \theta)^{5 / 3}}, & \text { Bo }<10 \\ \frac{\mathrm{Bo}}{\sqrt{\pi \operatorname{tg} \theta}}+\frac{3}{4 \sqrt{\mathrm{Bo}}}+\frac{3 \sqrt{\pi \operatorname{tg} \theta}}{32 \mathrm{Bo}^{5 / 4}}, & \text { Bo } \geqslant 10 .\end{cases}
$$

Здесь объединены два асимптотических выражения для $R$, полученные в [9] из граничного условия

$$
2 \frac{\operatorname{tg} \theta}{\mathrm{Bo} R}+\frac{1}{\pi R^{2}}+\frac{I_{0}(R \sqrt{\mathrm{Bo}}) \operatorname{tg} \theta}{\sqrt{\mathrm{Bo}} I_{1}(R \sqrt{\mathrm{Bo}})}=0
$$

при рассмотрении предельных случаев, когда Во $\rightarrow 0$ и Во $\rightarrow \infty$.

5.2. Параметры задачи. Пусть объем микрокапли равняется 1 мкл и макрокапли - 0.3 мл. Тогда характерные масштабы $L_{c}=1$ и $L_{c} \approx 6.7$ мм соответственно. Временной масштаб $T_{c}=1$ с для микрокапли и $T_{c} \approx 45$ с для макрокапли. Значения безразмерных параметров следующие: для меньшего объема жидкости Са $\approx 10^{-5}$, Во $\approx 0.1$ и для большего $-\mathrm{Ca} \approx 2 \cdot 10^{-6}$, $\mathrm{Bo} \approx 6$. В качестве подложки будем рассматривать поверхность с заданным углом смачивания $\theta \approx \pi / 6$. Из-за рассмотрения тонкого жидкого слоя считаем, что $\theta$ не зависит от объема капли.

5.3. Результаты расчетов. Результаты расчетов представлены на рис. 4. На графике видно, что форма поверхности макрокапли уплощенная, а равновесный профиль микрокапли больше по форме напоминает сферический сегмент (рис. 4, а). В зависимости от объема жидкости доминирует либо сила поверхностного натяжения (Во $<1$ ), либо сила тяжести (Во > 1), что и определяет форму двухфазной границы капли. 


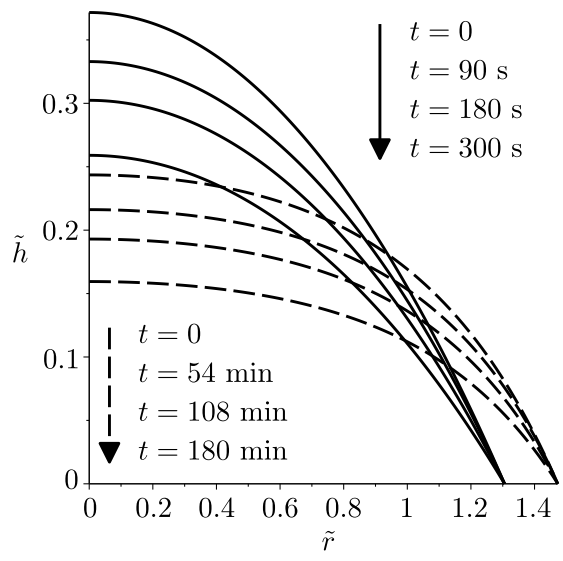

a

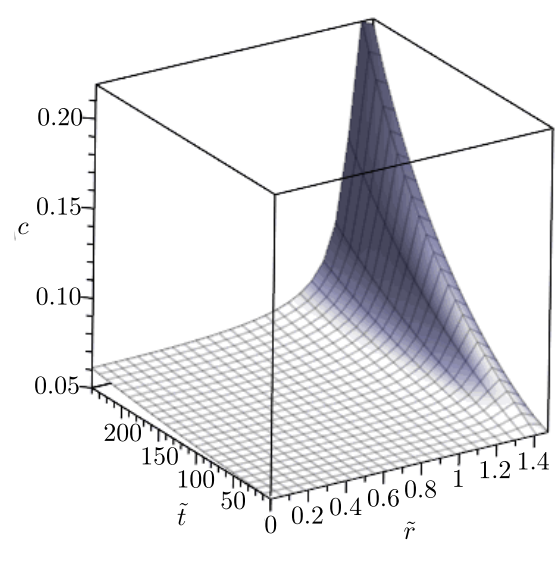

$b$

Рис. 4. Результаты расчета динамики коллоидной жидкости в микро- и макрокапле. Параметры расчета: $E=5 \cdot 10^{-5}, A=250, K=0.11 \pm 0.005$. На рисунке приведены: (а) толщина жидкого слоя (сплошные линии - микрокапля, пунктирные - макрокапля), (b) массовая доля коллоидных частиц (макрокапля)

[Figure 4. Results of calculating the dynamics of colloidal fluid in micro- and macrodrops. Calculation parameters: $E=5 \cdot 10^{-5}, A=250, K=0.11 \pm 0.005$. The figure shows: (a) the thickness of the liquid layer (solid lines-microdroplet, dashed lines-macrodrop), (b) the mass fraction of colloidal particles (macrodrop)]

Характер изменения массовой доли коллоидных частиц в макрокапле не отличается от случая с микрокаплей. Значение $c$ стремительно растет вблизи свободной границы капли «газ-жидкость-подложка» (рис. 4, b). Такое явление объясняется выносом частиц на периферию радиальным течением, возникающим в результате испарения жидкости. Таким образом, результаты расчетов качественно согласуются с экспериментальными наблюдениями эффекта «капли кофе» как в микро-, так и макрожидкостях коллоидных и молекулярных растворов [6,22].

5.4. Вывод. При математическом описании динамики жидкости в макрокаплях наряду с капиллярными силами необходимо учитывать силу тяжести. Массоперенос, возникающий в результате испарения, характерен для любого размера капель.

6. Численные методы. Для решения описанных выше задач выбран метод конечных разностей, реализованный в виде комплекса программ на языке Maple. Работа выполнена в коммерческой среде Maple 17. Пакет содержит ряд встроенных модулей для решения дифференциальных уравнений, кроме того, в нем имеются средства для программирования новых методов или модификации встроенных.

Общий вид рассматриваемых уравнений записывается как

$$
d \frac{\partial y}{\partial t}-a(x, t) \frac{\partial^{2} y}{\partial x^{2}}-b(x, t, y) \frac{\partial y}{\partial x}-c(x, t) y=f\left(x, t, y, y_{x}^{\prime}\right)
$$

где $d$ в каждом конкретном уравнении равно либо нулю, либо единице. Если порядок уравнения по пространству выше второго, то такое уравнение можно переписать в виде системы уравнений меньшего порядка. Это касается и про- 
извольного порядка по времени. Таким образом, при необходимости уравнение можно свести к общему виду (21). Также отметим, что коэффициент $b$ линеаризуется за счет введения вспомогательной функции $\gamma$. В п. 3 правая часть $f$ перестает зависеть от $y_{x}^{\prime}$ из-за использования приближения смазки, когда $l \approx 1$, а в п. 4 и 5 - из-за добавления вспомогательной величины $\omega$.

В п. 3 квазистационарная и нестационарная модели решаются численно с использованием Ө-схемы [23]. В отечественной литературе она больше известна как параметрическая двухслойная шеститочечная разностная схема [24]. Строится сеточная аппроксимация (21) в области $x \in[0, X] \times t \in[0, T]$

$$
d \frac{y_{i}^{j+1}-y_{i}^{j}}{\tau}=\theta \boldsymbol{\Lambda} y_{i}^{j+1}+(1-\theta) \boldsymbol{\Lambda} y_{i}^{j}+\theta f_{i}^{j+1}+(1-\theta) f_{i}^{j},
$$

где оператор

$$
\boldsymbol{\Lambda} y_{i}^{j}=\frac{a_{i}^{j}}{\xi^{2}}\left(y_{i+1}^{j}-2 y_{i}^{j}+y_{i-1}^{j}\right)+\frac{b_{i}^{j}}{2 \xi}\left(y_{i+1}^{j}-y_{i-1}^{j}\right)+c_{i}^{j} y_{i}^{j} .
$$

Здесь для сеточных функций используется обозначение $y_{i}^{j}=y\left(x_{i}, t_{j}\right)$, где $x_{i}=i \xi$-шаг по координате $\xi=X / N, N+1$ - количество узлов сетки по пространству, $i=0,1, \ldots, N, t_{j}=j \tau, \tau=T / M-$ шаг по времени, $M+1-$ количество узлов сетки по времени, $j=0,1, \ldots, M$. Соответственно, $y_{i \pm 1}^{j}=$ $=y\left(x_{i} \pm \xi, t_{j}\right), y_{i}^{j+1}=y\left(x_{i}, t_{j}+\tau\right)$. Также следует уточнить, что первые приближения для $b^{j+1}$ и $f^{j+1}$ берутся как $b^{j}$ и $f^{j}$ соответственно.

Для решения неустойчивой задачи из п. 4 и 5 описанный выше метод был модифицирован. В разностную аппроксимацию одного из уравнений системы введен регуляризатор [25]. Регуляризатор представляет собой слагаемое $\bar{R}\left(P_{i}^{j+1}-P_{i}^{j}\right) / \tau$, где $0<\bar{R} \ll 1$. Тогда сеточную аппроксимацию $(22)$ перепишем в виде

$$
(d+\bar{R}) \frac{y_{i}^{j+1}-y_{i}^{j}}{\tau}=\theta \boldsymbol{\Lambda} y_{i}^{j+1}+(1-\theta) \boldsymbol{\Lambda} y_{i}^{j}+\theta f_{i}^{j+1}+(1-\theta) f_{i}^{j}
$$

Таким образом, задача, в которой не используется следствие из теории смазки $l \approx 1$, решена с применением регуляризованной $\Theta$-схемы $(23)$.

Проблема сложности уравнений из-за некоторых нелинейных слагаемых устраняется за счет введения в систему вспомогательных величин $\omega$ и $\gamma$. Но в этом случае получаем некорректную задачу с неустойчивостью решения относительно малых изменений начальных условий. То есть некорректность в данном случае связана с плохой обусловленностью. Значение числа обусловленности стремится к бесконечности, так как у матрицы коэффициентов алгебраических уравнений не существует стандартной обратной матрицы. Дело в том, что эта матрица вырожденная, то есть ее определитель равен нулю. Поэтому решить задачу в такой постановке с помощью стандартного метода (схема с весами без регуляризатора) не получилось.

Расчеты проведены при значении параметра $\Theta=1$ (неявная схема конечных разностей с первым порядком аппроксимации по времени и вторым по пространству). Нелинейная система алгебраических уравнений на верхнем временном слое решается методом Ньютона. Выполнена проверка сходимости 
задачи по сетке. При расчетах используется сетка, количество узлов которой по пространству равняется 101. Значение шага по времени $\tau=\xi$, где $\xi$ - шаг по координате.

Заключение. Гидродинамические процессы в высыхающих каплях достаточно сложны, даже если не принимать во внимание фазовые переходы в капле и внешние возмущения формы поверхности. Ряд математических моделей использует те или иные упрощения системы уравнений Навье-Стокса. Во многих важных для практических приложений случаях достаточно рассматривать осесимметричные капли и ограничиться двумерной задачей.

При незначительных отклонениях формы капли от равновесной для медленных движений внутри капли можно пренебречь конвекционными потоками и рассматривать осредненное по глубине компенсационное движение. Процедура осреднения уравнений Навье-Стокса затруднена ввиду нелинейности самих уравнений. Поэтому различные авторы используют математические модели, зачастую без строгого их обоснования или описания процедуры вывода.

Примечательно, что такие модели часто дают хорошее количественное совпадение с некоторыми экспериментами. В статье проводится сравнение осредненной по глубине капли квазистационарной модели и нестационарной одномерной модели, предложенной ранее авторами, с экспериментальными данными. Показано, что в рассматриваемом диапазоне параметров обе модели дают удовлетворительные результаты для медленного испарения, но нестационарная модель лучше описывает финальную стадию процесса.

Упрощенный подход может применяться и к микрокаплям, и к макрокаплям. Основное отличие при этом наблюдается в форме свободной поверхности жидкости. А от ее вязкости зависит скорость испарения [5, 20] и компенсационного течения. Для моделирования процессов при быстром испарении и в случае значительных градиентов температуры необходимы более сложные математические модели рассматриваемых явлений. Впрочем, как и для случаев повышения вязкости раствора из-за дегидратации, а также дальнейшего фазового превращения золя в гель.

Особенность предложенной модели заключается в совместном учете влияния вязких, гравитационных и капиллярных сил на массоперенос в высыхающих каплях и пленках различных размеров. Для решения плохо обусловленной задачи о высыхающей капле (пленке) предложена и реализована регуляризованная $\Theta$-схема, являющаяся модификацией параметрической двухслойной шеститочечной разностной схемы. Практическая значимость заключается в возможности ее применения с целью получения устойчивых решений для данного класса нелинейных уравнений. На основе предложенной модификации численной схемы разработан комплекс программ, реализующих алгоритмы расчета скорости радиальных течений, динамики границы «жидкостьгаз» и эволюции массовой доли частиц в процессе испарения. Предложен способ получения кольцевых структур из микро- и наночастиц методом испарительной литографии. Описана геометрия маски, закрывающей ячейку коллоидной жидкости. Численные результаты показали, что массовая доля частиц увеличивается значительно под кольцевыми отверстиями в процессе высыхания. Особенность метода заключается в простоте, дешевизне и применимости практически к любой подложке без предварительной обработки. 
Конкурирующие интересы. Мы не имеем конкурирующих интересов.

Авторский вклад и ответственность. Все авторы принимали участие в разработке концепции статьи и в написании рукописи. Авторы несут полную ответственность за предоставление окончательной рукописи в печать. Окончательная версия рукописи была одобрена всеми авторами.

Финансирование. Работа выполнена при финансовой поддержке РФФИ (проект № 16-38-00439_мол_а).

\section{Библиографический список}

1. Sefiane K. Patterns from drying drops // Adv. Coll. Inter. Sci., 2014. vol. 206. pp. 372-381. doi: 10.1016/j.cis.2013.05.002.

2. Routh A. F. Drying of thin colloidal films // Rep. Prog. Phys., 2013. vol. 76, no. 4, 046603. doi: 10.1088/0034-4885/76/4/046603.

3. Harris D. J., Hu H., Conrad J. C., Lewis J. A. Patterning colloidal films via evaporative lithography// Phys. Rev. Lett., 2007. vol.98, no. 14, 148301. doi:10.1103/PhysRevLett. 98.148301.

4. Кухтевич И. В., Букатин А. С., Мухин И. С., Евстрапов А. А. Микрофлюидные чипы для исследования биологических объектов методами микроскопии высокого разрешения // Научно-технический вестник информационных технологий, механики и оптики, 2012. Т. 77, № 1. C. 111-115.

5. Rieger B., van den Doel L. R., van Vliet L. J. Ring formation in nanoliter cups: quantitative measurements of flow in micromachined wells // Phys. Rev. E, 2003. vol. 68, no. 3, 036312. doi: 10.1103/PhysRevE.68.036312.

6. Deegan R. D., Bakajin O., Dupont T. F., Huber G., Nagel S. R., Witten T. A. Contact line deposits in an evaporating drop// Phys. Rev. E, 2000. vol.62, no. 1. pp. 756-765. doi: 10. 1103/PhysRevE.62.756.

7. Fischer B. J. Particle convection in an evaporating colloidal droplet// Langmuir, 2002. vol. 18, no. 1. pp. 60-67. doi: 10.1021/la015518a.

8. Diana A., Castillo M., Brutin D., Steinberg T. Sessile drop wettability in normal and reduced gravity // Microgravity Sci. Technol., 2012. vol. 24, no. 3. pp. 195-202. doi: 10.1007/ s12217-011-9295-0.

9. Bartashevich M. V., Kuznetsov V. V., Kabov O. A. Gravity effect on the axisymmetric drop spreading // Microgravity Sci. Technol., 2010. vol. 22, no. 1. pp. 107-114. doi: 10.1007/ s12217-009-9153-5.

10. Коновалов В. И., Пахомов А. Н., Пахомова Ю. В. Геометрия, циркуляция и тепломассоперенос при испарении капли на подложке // Вестник ТГТУ, 2011. Т. 17, № 2. C. $371-387$.

11. Tarasevich Y. Y., Vodolazskaya I. V., Isakova O. P., Abdel Latif M. S. Evaporation-induced flow inside circular wells: analytical results and simulations // Microgravity Sci. Technol., 2009. vol. 21 (Suppl. 1). pp. 39-44. doi: 10.1007/s12217-009-9109-9.

12. Колегов К. С. Формирование кольцевых структур в высыхающей под шаблоном пленке коллоидного раствора // Вестн. ЮУрГУ. Сер. Матем. моделирование и программирование, 2014. Т. 7, № 1. С. 24-33. doi : 10.14529/mmp140103.

13. Колегов К. С., Лобанов А. И. Математическое моделирование динамики жидкости в испаряющейся капле с учетом капиллярных и гравитационных сил // Вестник РУДН. Серия: Математика, информатика, физика, 2014. №2. С. 375-380.

14. Колегов К. С. Сравнение квазистационарной и нестационарной математических моделей течений в испаряющейся капле с учетом вязкости // Вестн. Удмуртск. ун-та. Матем. Мех. Компъют. науки, 2014. №3. С. 110-122. doi: 10.20537/vm140310.

15. Колегов К. С., Лобанов А. И. Сравнение квазистационарной и нестационарной математических моделей течений в испаряющейся капле // Компъютерные исследования и моделирование, 2012. Т. 4, № 4. С. 811-825. 
16. Kaneda M., Takao Y., Fukai J. Thermal and solutal effects on convection inside a polymer solution droplet on a substrate // Int. J. Heat Mass Transfer, 2010.. vol. 53, no. 21-22. pp. 4448-4457. doi: 10.1016/j.ijheatmasstransfer.2010.06.049.

17. Jung Y., Kajiya T., Yamaue T., Doi M. Film formation kinetics in the drying process of polymer solution enclosed by bank// Jpn. J. Appl. Phys., 2009. vol. 48, no. 3, 031502. doi: 10.1143/JJAP. 48.031502.

18. Ehrhard P., Davis S. H. Non-isothermal spreading of liquid drops on horizontal plates // J. Fluid Mech., 1991. vol. 229. pp. 365-388. doi: 10.1017/S0022112091003063.

19. Cahile M., Benichou O., Cazabat A. M. Evaporating droplets of completely wetting liquids // Langmuir, 2002. vol. 18, no. 21. pp. 7985-7990. doi: 10.1021/1a020231e.

20. Hamamoto Y., Christy J. R. E., Sefiane K. Order-of-magnitude increase in flow velocity driven by mass conservation during the evaporation of sessile drops // Phys. Rev. E, 2011. vol. 83, 051602. doi: 10.1103/PhysRevE.83.051602.

21. Parneix C., Vandoolaeghe P., Nikolayev V. S., Quere D., Li J., Cabane B. Dips and rims in dried colloidal films // Phys. Rev. Lett., 2010. vol.105, no. 26, 266103. doi: 10.1103/ PhysRevLett.105.266103.

22. Bodiguel H., Leng J. Imaging the drying of a colloidal suspension // Soft Matter., 2010. vol. 6, no. 21. pp. 5451-5460. doi: 10.1016/j.cep.2012.07.005.

23. Stuart A. M., Peplow A. T. The Dynamics of the theta method// SIAM J. Sci. and Stat. Comput., 1991. vol. 12, no. 6. pp. 1351-1372. doi: 10.1137/0912074.

24. Самарский А. А., Гулин А. В. Численные методы. М.: Наука, 1989. 432 с.

25. Самарский А. А., Вабищевич П. Н. Разностные схемы для неустойчивых задач// Maтем. моделирование, 1990. Т. 2, №11. С. 89-98.

26. Okuzono T., Kobayashi M., Doi M. Final shape of a drying thin film // Phys. Rev. E, 2009. vol. 80, no. 2, 021603. doi: 10.1103/PhysRevE.80.021603.

27. Tarasevich Y. Y., Vodolazskaya I. V., Isakova O. P. Desiccating colloidal sessile drop: dynamics of shape and concentration // Colloid Polym. Sci., 2011. vol. 289, no. 9. pp. 1015-1023. doi : $10.1007 / \mathrm{s} 00396-011-2418-8$.

28. Maki K. L., Kumar S. Fast Evaporation of Spreading Droplets of Colloidal Suspensions // Langmuir, 2011. vol. 27, no. 18. pp. 11347-11363. doi: 10.1021/la202088s.

29. Barash L. Yu., Bigioni T. P., Vinokur V. M., Shchur L. N. Evaporation and fluid dynamics of a sessile drop of capillary size// Phys. Rev. E, 2009. vol. 79, no. 4, 046301. doi: 10.1103/ PhysRevE.79.046301.

30. Mollaret R., Sefiane K., Christy J. R. E., Veyret D. Experimental and Numerical Investigation of the Evaporation into Air of a Drop on a Heated Surface// Chem. Eng. Res. Design, 2004. vol. 82, no. 4. pp. 471-480. doi : 10.1205/026387604323050182.

31. Гордеева В. Ю., Люшнин А. В. Особенности испарения тонкого слоя воды в присутствии растворимого сурфактанта // ЖTФ, 2014. Т. 84, № 5. С. 28-34.

32. Lebedev-Stepanov P., Efimov S., Kobelev A. $012004 / /$ J. Phys. Conf. Series, 2017. vol. 925, no. 1. doi : 10.1088/1742-6596/925/1/012004.

33. Tarasevich Yu. Yu., Vodolazskaya I. V., Sakharova L. V. Mathematical modeling of pattern formation caused by drying of colloidal film under a mask // Eur. Phys. J. E., 2016. vol. 39, no. 2, 26. doi: 10.1140/epje/i2016-16026-5. 
MSC: 76T20, 76R99, 74M25, 82D80

\title{
Numerical study of mass transfer in drop and film systems using a regularized finite difference scheme in evaporative lithography
}

\author{
K. S. Kolegov ${ }^{1,2}$, A. I. Lobanov ${ }^{3}$ \\ 1 Astrakhan State University, \\ 20a, Tatishchev st., Astrakhan, 414056, Russian Federation. \\ 2 Volga State University of Water Transport \\ (Caspian Institute of Sea and River Transport the Branch of VSUWT), \\ 6, Nikolskaya st., Astrakhan, 414000, Russian Federation. \\ 3 Moscow Institute of Physics and Technology (State University), \\ 9, Inststitutskii per., Dolgoprudny, Moscow region, 141700, Russian Federation.
}

\begin{abstract}
Mass transfer in drying drops and films is interesting with practical point of view, since it is used in problems of evaporative lithography. Compensatory flows arise when conditions of nonuniform evaporation from the surface of the liquid layer are created and move colloidal particles in the region of fast evaporation. This makes it possible to obtain micro- and nanostructures of the required shape on a solid surface. Nonstationary model of mass transfer in drops and films is described in this paper. Feature of the model is to jointly take into account viscous, gravitational and capillary forces. To solve the unstable discrete problem on drying drop (film), a regularized finite difference scheme is proposed. A computer algorithm is developed on the basis of this scheme. We present a way of obtaining ring structures by using evaporative lithography method that based on the results of the computational experiments.
\end{abstract}

Keywords: mass transfer, drops, films, evaporative lithography.

Received: $10^{\text {th }}$ January, $2018 /$ Revised: $15^{\text {th }}$ May, $2018 /$

Accepted: $11^{\text {th }}$ June, $2018 /$ First online: $27^{\text {th }}$ June, 2018

\section{Research Article}

(이 (i) The content is published under the terms of the Creative Commons Attribution 4.0 International License (http://creativecommons.org/licenses/by/4.0/)

Please cite this article in press as:

Kolegov K. S., Lobanov A. I. Numerical study of mass transfer in drop and film systems using a regularized finite difference scheme in evaporative lithography, Vestn. Samar. Gos. Tekhn. Univ., Ser. Fiz.-Mat. Nauki [J. Samara State Tech. Univ., Ser. Phys. Math. Sci.], 2018, vol. 22, no. 2, pp. 344-363. doi:10.14498/vsgtu1601 (In Russian).

Authors' Details:

Konstantin S. Kolegov (1) http://orcid.org/0000-0002-9742-1308

Junior Research Fellow; Lab. of Mathematical Modeling and Information Technologies in Science and Education ${ }^{1}$; Senior Lecturer; Dept. of Mathematical and Natural Sciences Disciplines ${ }^{2}$; e-mail: konstantin.kolegov@asu . edu .ru

Alexey I. Lobanov (1) http://orcid.org/0000-0003-0391-3289

Dr. Phys. \& Math. Sci.; Professor; Dept. of Computational Mathematics ${ }^{3}$;

e-mail: alexey@crec.mipt.ru 
Competing interests. We have no competing interests.

Authors' contributions and responsibilities. Each author has participated in the article concept development and in the manuscript writing. The authors are absolutely responsible for submitting the final manuscript in print. Each author has approved the final version of manuscript.

Funding. The reported study was funded by RFBR according to the research project no. 16-38-00439_mol_a.

\section{References}

1. Sefiane K. Patterns from drying drops, Adv. Coll. Inter. Sci., 2014, vol. 206, pp. 372-381. doi: 10.1016/j.cis.2013.05.002.

2. Routh A. F. Drying of thin colloidal films, Rep. Prog. Phys., 2013, vol. 76, no. 4, 046603. doi : 10.1088/0034-4885/76/4/046603.

3. Harris D. J., Hu H., Conrad J. C., Lewis J. A. Patterning colloidal films via evaporative lithography, Phys. Rev. Lett., 2007, vol.98, no. 14, 148301. doi : 10.1103/PhysRevLett. 98. 148301.

4. Kukhtevich I. V., Bukatin A. S., Mukhin I. S., Evstrapov A. A. Microfluidic chips for the study of biological objects using high-resolution microscopy, Nauchno-tekhnicheskii vestnik informatsionnykh tekhnologii, mekhaniki $i$ optiki, 2012, vol.77, no. 1, pp. 111-115 (In Russian).

5. Rieger B., van den Doel L. R., van Vliet L. J. Ring formation in nanoliter cups: quantitative measurements of flow in micromachined wells, Phys. Rev. E, 2003, vol.68, no. 3, 036312. doi : 10.1103/PhysRevE.68.036312.

6. Deegan R. D., Bakajin O., Dupont T. F., Huber G., Nagel S. R., Witten T. A. Contact line deposits in an evaporating drop, Phys. Rev. E, 2000, vol.62, no.1, pp. 756-765. doi: 10. 1103/PhysRevE.62.756.

7. Fischer B. J. Particle convection in an evaporating colloidal droplet, Langmuir, 2002, vol. 18, no. 1, pp. 60-67. doi: 10.1021/la015518a.

8. Diana A., Castillo M., Brutin D., Steinberg T. Sessile drop wettability in normal and reduced gravity, Microgravity Sci. Technol., 2012, vol.24, no.3, pp. 195-202. doi:10.1007/ s12217-011-9295-0.

9. Bartashevich M. V., Kuznetsov V. V., Kabov O. A. Gravity effect on the axisymmetric drop spreading, Microgravity Sci. Technol., 2010, vol.22, no. 1, pp. 107-114. doi: 10.1007/ s12217-009-9153-5.

10. Konovalov V. I., Pakhomov A. N., Pakhomova Yu. V. Geometry, Circulation and Heat and Mass Transfer in Evaporation of Drop on the Substrate, Transactions of the TSTU, 2011, vol. 17, no. 2, pp. 371-387 (In Russian).

11. Tarasevich Y. Y., Vodolazskaya I. V., Isakova O. P., Abdel Latif M. S. Evaporation-induced flow inside circular wells: analytical results and simulations, Microgravity Sci. Technol., 2009, vol. 21 (Suppl. 1), pp. 39-44. doi : 10.1007/s12217-009-9109-9.

12. Kolegov K. S. Formation of Ring Structures in a Drying under the Mask Film of Colloidal Solution, Bulletin of the South Ural State University, Series: Mathematical Modelling, Programming and Computer Software, 2014, vol.7, no. 1, pp. 24-33 (In Russian). doi: $10.14529 / \mathrm{mmp} 140103$.

13. Kolegov K. S., Lobanov A. I. Mathematical Modeling of Fluid Dynamics in Evaporating Drop with Taking into Account Capillary and Gravitational Forces, Vestnik Rossiiskogo universiteta druzhby narodov. Seriya: Matematika. Informatika. Fizika, 2014, no. 2, pp. 375380 (In Russian).

14. Kolegov K. S. Comparison of quasisteady and nonsteady mathematical models of fluid flow in evaporating drop with due regard for the viscosity, Vestn. Udmurtsk. Univ. Mat. Mekh. Komp. Nauki, 2014, no. 3, pp. 110-122 (In Russian). doi: 10.20537/vm140310. 
15. Kolegov K. S., Lobanov A. I. Comparing of a quasisteady and nonsteady mathematical models of fluid flow in evaporating drop, Computer Research and Modeling, 2012, vol. 4, no. 4, pp. 811-825 (In Russian).

16. Kaneda M., Takao Y., Fukai J. Thermal and solutal effects on convection inside a polymer solution droplet on a substrate, Int. J. Heat Mass Transfer, 2010., vol. 53, no. 21-22, pp. 4448-4457. doi: 10.1016/j.ijheatmasstransfer.2010.06.049.

17. Jung Y., Kajiya T., Yamaue T., Doi M. Film formation kinetics in the drying process of polymer solution enclosed by bank, Jpn. J. Appl. Phys., 2009, vol. 48, no. 3, 031502. doi : 10.1143/JJAP. 48.031502.

18. Ehrhard P., Davis S. H. Non-isothermal spreading of liquid drops on horizontal plates, J. Fluid Mech., 1991, vol. 229, pp. 365-388. doi: 10.1017/S0022112091003063.

19. Cahile M., Benichou O., Cazabat A. M. Evaporating droplets of completely wetting liquids, Langmuir, 2002, vol. 18, no. 21, pp. 7985-7990. doi: 10.1021/la020231e.

20. Hamamoto Y., Christy J. R. E., Sefiane K. Order-of-magnitude increase in flow velocity driven by mass conservation during the evaporation of sessile drops, Phys. Rev. E, 2011, vol. 83, 051602. doi: 10.1103/PhysRevE. 83.051602.

21. Parneix C., Vandoolaeghe P., Nikolayev V. S., Quere D., Li J., Cabane B. Dips and rims in dried colloidal films, Phys. Rev. Lett., 2010, vol.105, no. 26, 266103. doi: 10.1103/ PhysRevLett. 105.266103.

22. Bodiguel H., Leng J. Imaging the drying of a colloidal suspension, Soft Matter., 2010, vol. 6, no. 21, pp. 5451-5460. doi: 10.1016/j.cep. 2012.07.005.

23. Stuart A. M., Peplow A. T. The Dynamics of the theta method, SIAM J. Sci. and Stat. Comput., 1991, vol. 12, no. 6, pp. 1351-1372. doi: 10.1137/0912074.

24. Samarskii A. A., Gulin A. V. Chislennye metody [Numerical Methods]. Moscow, Nauka, 1989, 432 pp. (In Russian)

25. Samarskii A. A., Vabishchevich P. N. Difference schemes for non-stable problems, Matem. Mod., 1990, vol. 2, no. 11, pp. 89-98 (In Russian).

26. Okuzono T., Kobayashi M., Doi M. Final shape of a drying thin film, Phys. Rev. E, 2009, vol. 80, no. 2, 021603. doi: 10.1103/PhysRevE.80.021603.

27. Tarasevich Y. Y., Vodolazskaya I. V., Isakova O. P. Desiccating colloidal sessile drop: dynamics of shape and concentration, Colloid Polym. Sci., 2011, vol. 289, no. 9, pp. 1015-1023. doi: 10.1007/s00396-011-2418-8.

28. Maki K. L., Kumar S. Fast Evaporation of Spreading Droplets of Colloidal Suspensions, Langmuir, 2011, vol. 27, no. 18, pp. 11347-11363. doi: 10.1021/la202088s.

29. Barash L. Yu., Bigioni T. P., Vinokur V. M., Shchur L. N. Evaporation and fluid dynamics of a sessile drop of capillary size, Phys. Rev. E, 2009, vol.79, no. 4, 046301. doi: 10.1103/ PhysRevE. 79.046301.

30. Mollaret R., Sefiane K., Christy J. R. E., Veyret D. Experimental and Numerical Investigation of the Evaporation into Air of a Drop on a Heated Surface, Chem. Eng. Res. Design, 2004, vol. 82, no. 4, pp. 471-480. doi : 10.1205/026387604323050182.

31. Gordeeva V. Yu., Lyushnin A. V. Peculiarities of evaporation of a thin water layer in the presence of a solvable surfactant, Tech. Phys., 2014, vol.59, no. 5, pp. 656-662. doi: 10. 1134/S1063784214050090.

32. Lebedev-Stepanov P., Efimov S., Kobelev A. 012004, J. Phys. Conf. Series, 2017, vol. 925, no. 1. doi : 10.1088/1742-6596/925/1/012004.

33. Tarasevich Yu. Yu., Vodolazskaya I. V., Sakharova L. V. Mathematical modeling of pattern formation caused by drying of colloidal film under a mask, Eur. Phys. J. E., 2016, vol. 39, no. 2, 26. doi: 10.1140/epje/i2016-16026-5. 